\title{
Towards Spectrum-Programmable, Mesh-Enabled Mobile Xhaul through Reconfigurable WDM Overlay in Fully-Passive Networks
}

\author{
Bernhard Schrenk, Member, IEEE, Thomas Lorünser, Thomas Zemen, Senior Member, IEEE \\ AIT Austrian Institute of Technology, Dept. Digital Safety\&Security / Optical Quantum Technology \\ Donau-City Strasse 1, A-1220 Vienna, Austria \\ Tel: +43 50550-4131,Fax:+43 50550-4190,e-mail: bernhard.schrenk@ait.ac.at
}

\begin{abstract}
A scheme for flexible networking is presented for the mobile front- and/or backhaul. Reconfigurable add/drop nodes are employed at the fibre plant to enable dynamic bandwidth allocation at wavelength level, as well as feeder protection and the formation of virtual optical networks. With these functionalities available capacity at the mobile backhaul can be distributed among datacentres of cloud radio access network (RAN) in order to adapt to actual load conditions at the baseband units (BBU). Moreover, the ability to form virtual point-to-point links between BBUs belonging to different cloud-RANs as well as between remote radio heads and baseband units of different cloud-RAN entities can optimize the utilization of network resources as a whole. The key to do so is a mobile front-/backhaul leveraged by reconfigurable optical add/drop multiplexers (ROADM). To the first time energy harvesting is applied to provide this functionality while retaining a fully-passive optical network.
\end{abstract}

Keywords: Fibre-optic communications, Passive optical networks, ROADM, Radio access networks, Mobile fronthaul, Mobile backhaul

\section{INTRODUCTION}

The leap towards $5 \mathrm{G}$ radio technology puts optical access and the metro aggregation network once more into the spotlight. In order to enable this next generation of the broadband ecosystem, wireless and optical access technologies have to be advanced hand-in-hand. Cloud-based processing of radio signals through the so-called Cloud-RAN paradigm, which is sketched in Fig. 1, enables a more energy-efficient radio access network (RAN) through advanced coordination and interference management schemes [1]-[2]. However, a high capacity link is required between the remote radio head (RRH) at the antenna site and the consolidated baseband unit (BBU). While the backhaul between BBUs and the central office (CO) is already realized through optics, lightwave communication can also be an enabler for massive fronthaul capacity between RRH and BBU [3]. The maximum reach of the fronthaul is determined by the latency requirements set for the link between radio unit and BBU. Typically accepted fronthaul round-trip times of $300 \mu$ s correspond to a reach of $40 \mathrm{~km}$. On the other hand, the reach of extended PONs can be anticipated with in-between 60 and $100 \mathrm{~km}$ and is therefore about twice as large. This means that per $\mathrm{CO}$ a number of at least $4 \mathrm{BBU}$ pools can be expected in case of applying the cloud-RAN scheme. Spectral load balancing among the BBU pools and path protection will be a paramount for the mobile backhaul within metro-access PONs. At the same time the fronthaul will require highly dynamic resource assignment so to adapt the spectral bandwidth per RRH as users shift between geographic hotspots. The use of mm-wave and MIMO techniques is expected to fuel the need for WDM overlay since equivalent CPRI data rates beyond $100 \mathrm{~Gb} / \mathrm{s}$ are anticipated per RRH site in case of LTE and carrier aggregation. Inter-BBU communication through virtual PON routing capability can facilitate X2 traffic for the handover of mobile users between the BBUs and the extension of particular BBU pools for mobile users not requiring low latency. This avoids to route traffic through the $\mathrm{CO}$ and saves therefore power consumption as o/e/o conversions and data processing are bypassed while a shorter lightpath is offered at the same time.
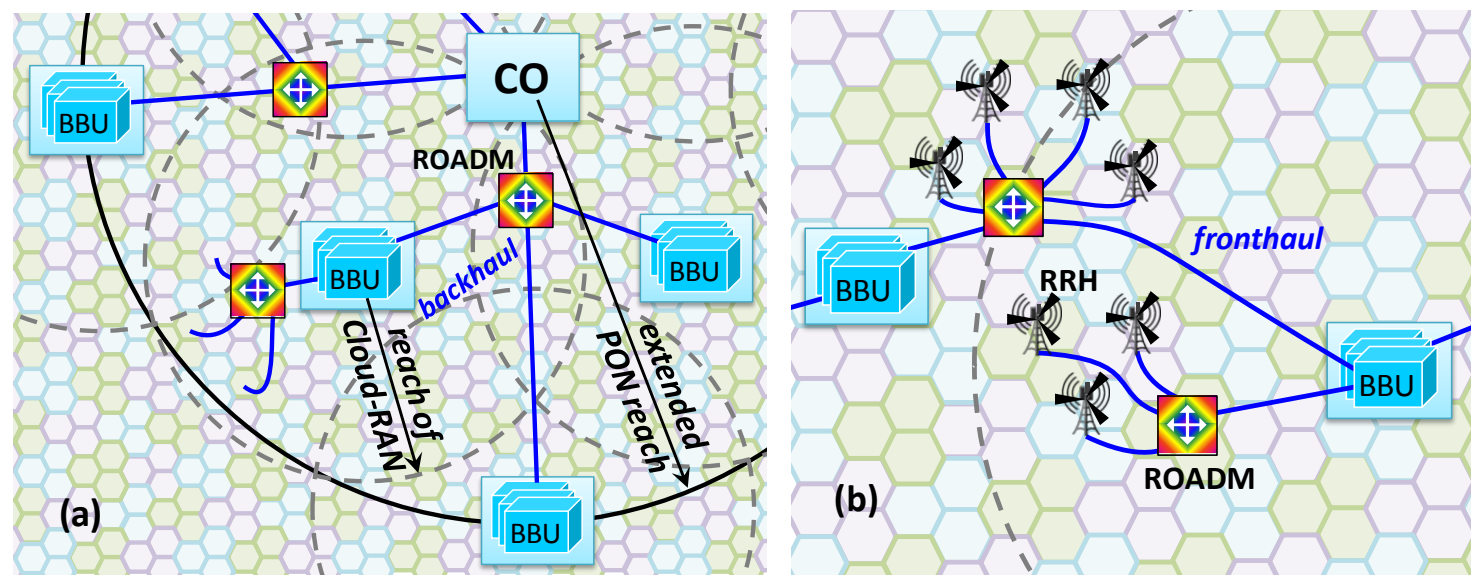

Figure 1. Flexibility brought by fully-passive reconfigurable nodes to (a) mobile backhaul and (b) fronthaul. 
In this paper we provide a fully-passive ROADM concept as possible solution to dynamic resource assignment at the front-/backhaul and for the formation of virtual networks between BBU sites while providing resiliency features on top of the previous. Needless to say, conventional ROADMs as found in metro-core networks [4] do not bring the required cost and energy efficiency for use in metro-access PONs. On the contrary, the proposed node concept offers a similar degree of flexible networking functionality and takes advantage of energy scavenging techniques in order to obtain the required energy for resource or lightpath switching [5], which is supported and coordinated through an integrated control plane interface [6]. In this way flexible metro-access PONs are enabled while retaining their passiveness, meaning low capital and operating expenditures as no local electrical power supplies are required at the optical distribution network.

\section{LEVERAGING FRONT-/BACKHAUL FLEXIBILITY: THE FULLY-PASSIVE ROADM}

The architecture of the proposed ROADM is presented in Fig. 2. The node is designed for two feeder and four drop ports. It can be used in conventional metro-access PONs or in context of mobile back- and fronthauling. The ROADM is intended to handle four wavebands which are each dedicated to one of the drop ports. Optical switching is exploited within the node to (a) provide resiliency functionality towards the feeder ports, (b) provide multi-casting of the wavebands to the drop ports, (c) establish lightpaths between the drop ports. These three functionalities can offer feeder/backhaul protection, dynamic spectrum allocation in these domains and virtual PON formation between BBU sites (Fig 1a) or between RRH and BBU sites for the purpose of switching RRHs at cloud-RAN boundaries towards underutilized BBUs (Fig 1b). In case of the presented ROADM a design using CWDM channels from 1550 to $1610 \mathrm{~nm}$ has been chosen. Within these CWDM wavebands a number of DWDM channels can be aggregated, which is in-line with WDM overlay as envisaged in NG-PON2.

Network protection is facilitated through a $2 \times 2$ switch $(r)$ based on latching micro-opto-electro-mechanic system (MOEMS) technology as recently demonstrated in fully-passive manner [5]. The economic and ecologic meaningful principle of PONs can be thereby retained despite the introduction of electrically actuated optical networking elements. Multi-casting of wavebands to the drop ports is achieved by routing wavebands $\Lambda_{i-1}$ and/or $\Lambda_{i+1}$ adjacent to the primary waveband $\Lambda_{i}$ of drop port $i$ to the multiplexer of this port. This functionality is enabled by a $2 \times 1$ MOEMS switch $(m)$ whereas the waveband extension for a particular tree port is set by four individual waveband switches $\left(\Lambda_{i}\right)$ that are each dedicated to one of the spectral bands $\Lambda_{1}$ to $\Lambda_{4}$. For example, if drop port 3 receiving $\Lambda_{3}$ shall be extended by $\Lambda_{2}$ and $\Lambda_{4}$, these extra wavebands are switched by the corresponding MOEMS switches to the CWDM multiplexer at drop port 3, which itself appears as port $i+1$ and $i-1$ for these wavebands. On the other hand, $\Lambda_{3}$ can be shared with drop ports 2 and 4 .

Finally, virtual PONs can be formed between the drop ports in order to link BBU sites for the purpose of load sharing. This functionality is supported through a loop configuration that incorporates the $i, m$ and $\Lambda_{i}$ MOEMS switches. For example, if a link between drop ports 3 and 1 shall be established, either waveband $\Lambda_{2}$ or $\Lambda_{4}$ can be used since both wavebands are (cyclic) adjacent to these drop ports, meaning that these wavebands connect to both ports through the CWDM multiplexers "inter" and "multi". The 50/50 split loss at the feeder-side input of the ROADM that is used to enable multi-cast functionality can be bypassed through the MOEMS switches $i$ and $m$ in case that multi-cast operation is not required at the same time.

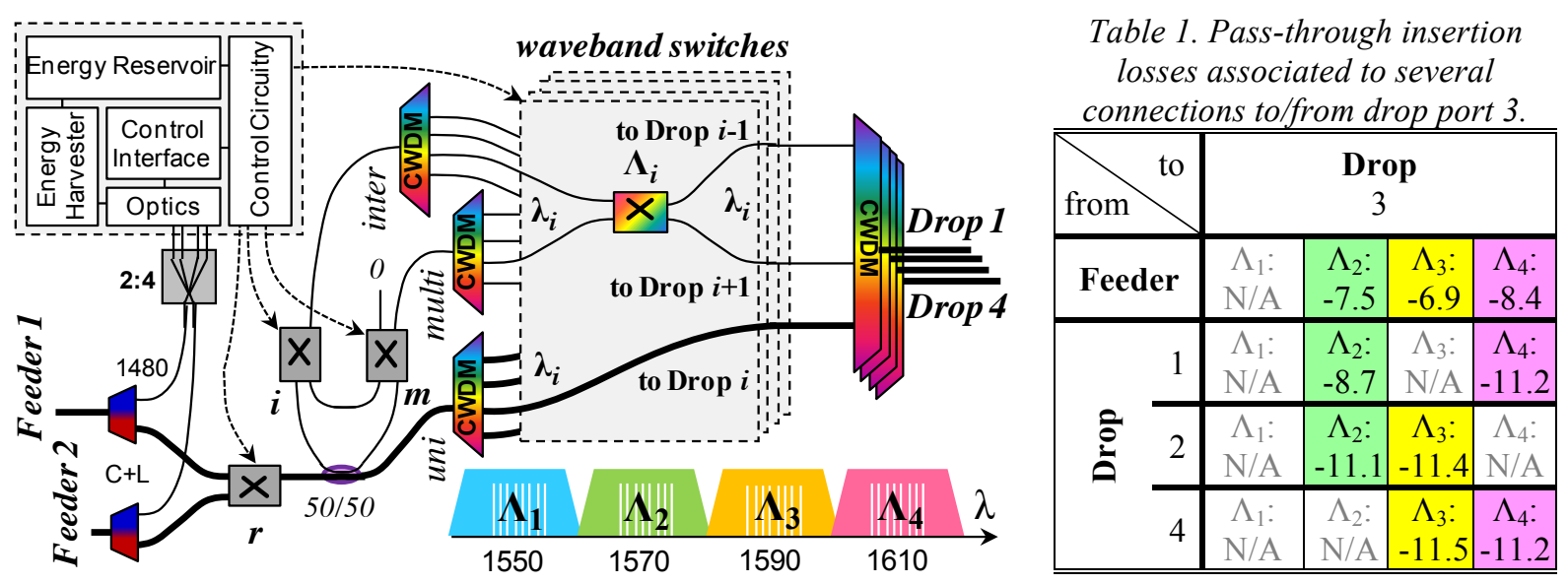

Figure 2. Fully-passive ROADM for flexible front-/backhauling.

A total number of seven $2 \times 2$ MOEMS switches and seven CWDM multiplexers is required in order to realize this $2 \times 4$ ROADM configuration with multicast, resiliency and inter-BBU networking functionalities. At utmost importance for the ROADM is a control circuitry solely fed by optical power. This low-power electronics module is identical to that reported in [6] and is able to handle control plane signals modulated onto the optical pump used for feeding the ROADM. Energy harvesting at low optical feed power levels of $-9 \mathrm{dBm}$ at $1480 \mathrm{~nm}$ is 
exploited to fill capacitive charge reservoirs with low leakage through a photovoltaic stack of four PIN diodes, followed by a capacitive boost-converter. A microcontroller with an average power consumption of $1.4 \mu \mathrm{A}$ at a supply of $1.6 \mathrm{~V}$ is used to handle control plane messages, the actuation of MOEMS switches and the power cycling within the module. Due to the latching nature of the switches there is no steady-state power consumption in between two reconfiguration steps besides the negligibly low consumption of the node controller.

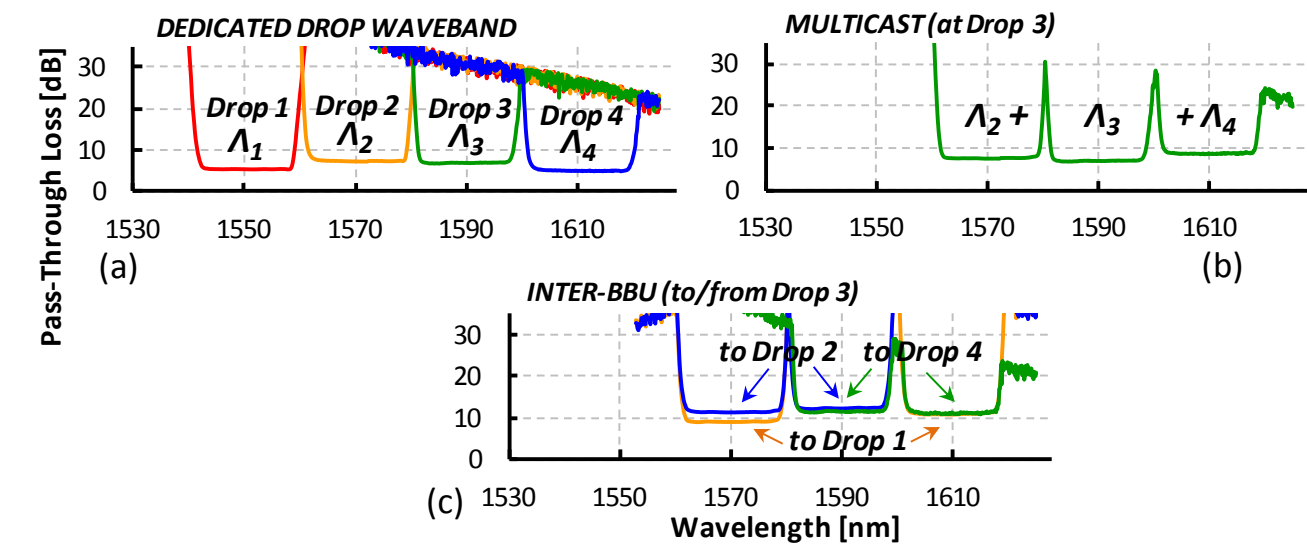

Figure 3. Transmission characteristics for the ROADM. (a) Dedicated waveband per drop port, (b) multicasting at drop port 3, (c) inter-BBU connections to and from drop port 3.

The transmission spectra between feeder and drop ports are shown in Fig. 3a while those between drop ports are presented in Fig. 3c. The spectral -3dB-bandwidth of a waveband is in average $16.8 \mathrm{~nm}$, which represents the assigned bandwidth per drop port. This spectral slice can be extended by use of adjacent wavebands up to more than $50 \mathrm{~nm}$. This is shown in Fig. $3 \mathrm{~b}$ for the particular case of drop port 3 that is then receiving the slices $\Lambda_{2}$ to $\Lambda_{4}$. The fibre-to-fibre pass-through loss for the ROADM is $8.4 \mathrm{~dB}$ in the worst-case. Inter-BBU transmission can be facilitated between this drop port and several other drop ports, which is evident from Fig. $3 \mathrm{c}$. The passthrough insertion loss increases to $11.5 \mathrm{~dB}$ in the worst case since the signal needs to traverse four CWDM multiplexers, one 50/50 coupler and four MOEMS switches. Table I summarizes the wavelength assignments and corresponding pass-through losses for several interconnections established for drop port 3.

\section{DYNAMIC SPECTRUM SWITCHING AND FORMATION OF VIRTUAL PONS}

The experimental setup for evaluating dynamic spectrum allocation among drop segments and the establishment of inter-BBU connections is presented in Fig. 4a. The fully passive ROADM is located between feeder and drop segments that connect to the OLTs and ONUs, respectively. In the context of mobile fronthauling, as introduced in Fig. 1, the ONUs can represent RRHs while the OLTs can stand for BBUs. Moreover, in case of mobile backhauling the ONUs can further represent BBUs while the OLTs would then be hosted at different COs.

Switching of spectral resources from the OLT is investigated by multiplexing and transmitting different signals towards the feeder: one comb in the C-band, one comb in the L-band and a single channel at $1610 \mathrm{~nm}$. The pump at $1480 \mathrm{~nm}$ that constitutes the optical feed and control plane signal for the ROADM is also added with a power of $3 \mathrm{dBm}$. In addition, one ONU is added per drop segment. These ONUs are emitting at different wavelengths, in particular at 1568,1569, 1571 and $1611 \mathrm{~nm}$. Measurements are taken at drop port 3 and BER measurements through the wavelength-tunable receiver at ONU-3. The feeder was chosen with $42.3 \mathrm{~km}$ of single-mode fibre while the drop segments were $2 \times 4.3 \mathrm{~km}$ long with a power splitting stage in between.

Reallocation of wavelength resources from the feeder to drop segment 3 is presented in Fig. 4b. Waveband $\Lambda_{3}$ is continuously associated to this drop segment and includes the upper L-band comb (A in Fig. 4b) transmitted by the OLT. Multicasting is then enabled through the corresponding MOEMS switch $m$ and waveband $\Lambda_{2}$ is appended to $\Lambda_{3}$. With this the lower portion of the L-band comb (B) and also the upper part of the C-band comb (C) is dropped to port 3. Note that waveband $\Lambda_{4}$ is not forwarded despite activated multicasting since it is by default associated to drop port 1 . As next reconfiguration step inter-BBU links are set up through actuating MOEMS switch $i$. Lightpaths between drop segments 1-3 and 3-4 are established at $1568 \mathrm{~nm}(\mathrm{E})$ and $1611 \mathrm{~nm}$ (D), respectively. This allows that BBUs at locations ONU-1 and ONU-4 can be virtually linked to the BBUs pooled at ONU-3. In the next step the inter-BBU link between drop segments 3-4 is exchanged by multicasting of waveband $\Lambda_{4}$ to drop segment 3 . This is made by flipping MOEMS switch $\Lambda_{4}$. As a consequence, the OLT channel at $1610 \mathrm{~nm}(\mathrm{~F})$ does now appear in the spectrum. This reconfiguration step also shows that not all functionalities can be provided at the same time, e.g., inter-BBU communication and multicasting of extension bands for WDM overlay that are also used for the purpose of facilitating inter-BBU traffic. Still it has to be recalled that the OLT channel at $1610 \mathrm{~nm}$ and several channels in waveband $\Lambda_{2}(\mathrm{~B}, \mathrm{C})$ originating from the OLT are also present at their designated drop ports 4 and 2, respectively. These extension wavelengths can be therefore shared by means of TDM among the drop segments. The last reconfiguration shown in Fig. 4b, which 


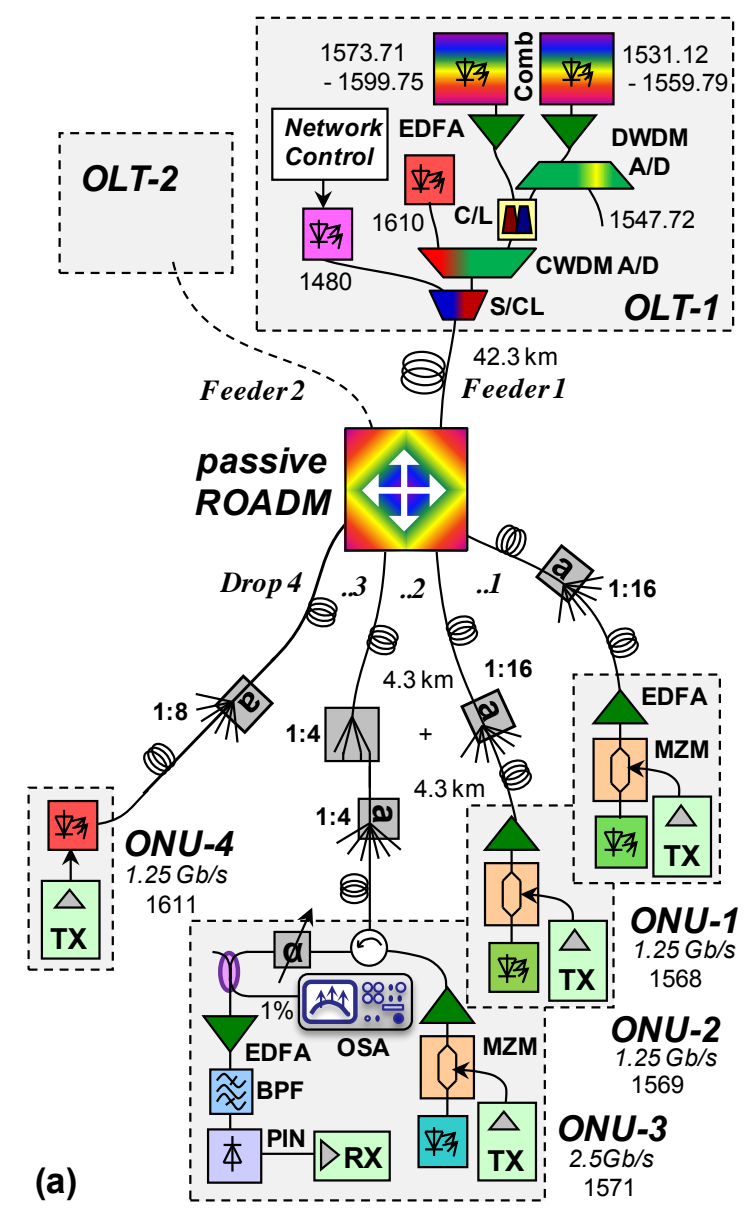

is to flip MOEMS switch $\Lambda_{2}$, leads to inter-BBU communication between drop segments 2-3 at the channel of $1569 \mathrm{~nm}(\mathrm{G})$ and depletes the energy reservoir. Similar to what was stated previously, this takes the opportunity to extend the feeder-side spectrum towards drop segment 3 by waveband $\Lambda_{2}(B, C)$ since several wavelengths within this spectral band are now intended to support inter-BBU traffic. However, inter-BBU traffic between drop segments 1-3 at the channel of $1568 \mathrm{~nm}(\mathrm{E})$ is not affected by this last operation. It shall be stressed that four reconfiguration steps can be facilitated before recharging the energy reservoir similar as multiple consecutive switching operations have been demonstrated in [5].

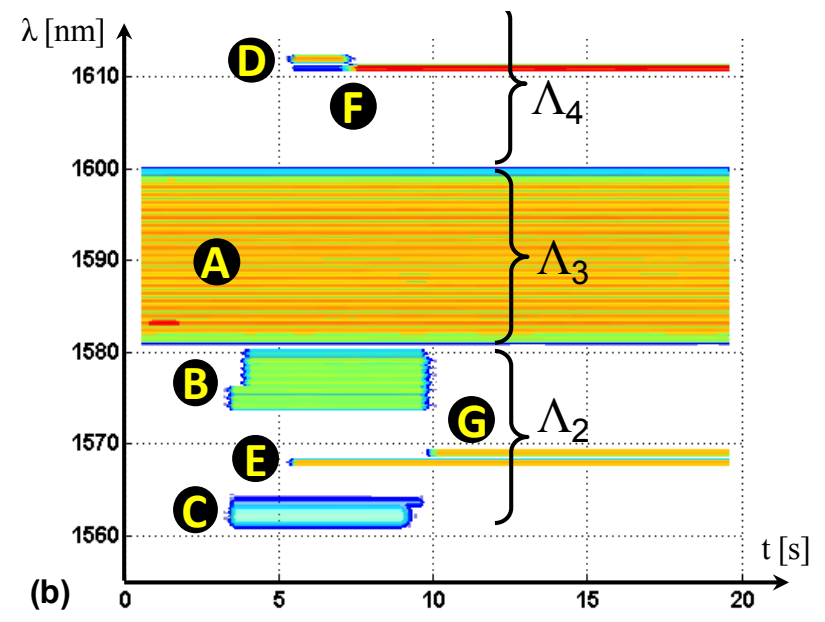

Figure 4. (a) Experimental setup and (b) re-allocation of spectral resources and lightpaths at drop port 3.

Finally, BER measurements have been carried out to evaluate if crosstalk from channels adjacent in space and wavelength raises error floors. For this purpose data transmission between the OLT and ONU-3 and between ONU-1, -2, -4 and ONU-3 has been evaluated at 1.25 and $2.5 \mathrm{~Gb} / \mathrm{s}$ in a back-to-back case and in presence of the ROADM. No penalty has been observed, which is attributed to the sufficiently high side-channel rejection for the CWDM multiplexers used in the ROADM and further due to the very low crosstalk ratio below $-54 \mathrm{~dB}$ between the ports of its $2 \times 2$ MOEMS switches [6].

\section{CONCLUSIONS}

A fully-passive node concept that avoids electrically-powered networking equipment in passive front- and backhaul links has been demonstrated. The proposed ROADM uses 4 CWDM wavebands and provides ondemand multicast functionality, resiliency and the option to establish virtual PONs between BBUs located in different cloud-RANs. With these a way for load balancing and dynamic bandwidth allocation is provided for the mobile front- and/or backhaul without compromising the passiveness of the optical network.

\section{ACKNOWLEDGEMENTS}

This work was supported by the European Commission through the FP7 Marie-Curie grant WARP-5 (n 333806).

\section{REFERENCES}

[1] C. I, et al:: Toward green and soft: A 5G perspective, IEEE Comm. Mag., vol. 52, pp. 66-73, Feb. 2014.

[2] P. Demestichas, et al.: 5G on the horizon: key challenges for the radio- access network, IEEE Veh. Technol. Mag., vol. 8, pp. 47-53, Sept. 2013.

[3] C. Liu, et al:: A Novel Multi-Service Small-Cell Cloud Radio Access Network for Mobile Backhaul and Computing Based on Radio-Over-Fiber Technologies, J. Lightwave Technol., vol. 31, pp. 2869-75, 2013.

[4] S. Gingeri, B. Basch, V. Shukla, R. Egorov, T. Xia: Flexible Architectures for Optical Transport Nodes and Networks, IEEE Comm. Mag., vol. 48, pp. 40-50, Jul. 2010.

[5] B. Schrenk, A. Poppe, M. Stierle, H. Leopold: Fully-Passive Optical Switch Introducing Dynamicity and Flexibility to Metro-Access, Phot. Technol. Lett., vol. 27, pp. 486-489, Mar. 2015.

[6] B. Schrenk, et al.: Fully-Passive Resiliency Switch for Agile PON Restoration, in Proc. OFC'15, Los Angeles, United States, Mar. 2015, paper W1J.6. 